\title{
An evolutionary sequence of expanding hydrogen shells in galaxy discs
}

\author{
M. Relaño ${ }^{1}$, J. E. Beckman ${ }^{2,3}$, O. Daigle ${ }^{4}$, and C. Carignan ${ }^{4}$
}

\author{
1 Dpto. Física Teórica y del Cosmos, Universidad de Granada, Avda. Fuentenueva s/n, 18071 Granada, Spain \\ e-mail: mrelano@ugr.es \\ 2 Instituto de Astrofísica de Canarias, C. Vía Láctea s/n, 38200 La Laguna, Tenerife, Spain \\ 3 Consejo Superior de Investigaciones Científicas (CSIC), Spain \\ e-mail: jeb@ll.iac.es \\ ${ }^{4}$ Observatoire du mont Mégantic, LAE, Université de Montréal, C. P. 6128 succ. centre ville, Montréal, Québec, H3C 3J7, Canada \\ e-mail: [odaigle; carignan]@ASTRO.UMontreal.CA
}

Received 13 June 2006 / Accepted 12 March 2007

\begin{abstract}
Aims. Large HI shells, with diameters of hundreds of pc and expansion velocities of $10-20 \mathrm{~km} \mathrm{~s}^{-1}$ have been detected in their hundreds in the Milky Way and are well observed features of local gas rich galaxies. These shells could well be predicted as a result of the impact of OB associations on the ISM, but doubt has been cast on this scenario by the apparent absence of OB stars close to the centres of a large fraction of these shells in recent observations of the SMC. Here we present observational evidence within an energetically consistent framework which strongly supports the scenario in which OB associations do produce the giant HI shells.

Methods. Using Fabry-Perot scanned $\mathrm{H} \alpha$ emission line mapping of nearby galaxy discs, we have detected, in all the H II regions where the observations yield sufficient angular resolution and $\mathrm{S}: \mathrm{N}$ ratio, dominant $\mathrm{H} \alpha$ shells with radii a few tens of pc, expanding at velocities of 50-100 $\mathrm{km} \mathrm{s}^{-1}$, and with gas masses of $10^{4}-10^{5} M_{\odot}$. In previous studies, we found that stellar winds alone can account for the energetics of most of the $\mathrm{H} \alpha$ shells, which form initially before the stars explode as SNe. We have applied a simple dynamically consistent framework in which we can extrapolate the properties of the observed $\mathrm{H} \alpha$ shells to a few $10^{7} \mathrm{yr}$ after the formation of the OB stars. The framework includes the dynamical inputs of both winds and SNe on the surrounding ISM. The results give quantitative statistical support to the hypothesis that the $\mathrm{H} \alpha$ emitting shells are generic progenitors of the HI shells.

Results. The results are in good agreement with the ranges of masses $\left(\sim 10^{6} M_{\odot}\right)$, velocities (up to $\left.\sim 20 \mathrm{~km} \mathrm{~s}^{-1}\right)$, and diameters (up to $\sim 500 \mathrm{pc}$ ) of representative HI shells observed in nearby galaxies. The combined effects of stellar winds, acting during the first few $10^{6} \mathrm{yr}$, and SN explosions, "switching on" subsequently, are required to yield the observed parameters.
\end{abstract}

Key words. ISM: H II regions - ISM: kinematics and dynamics - ISM: bubbles - galaxies: general

\section{Introduction}

The presence of neutral hydrogen shells in the gas of disc galaxies is a widely known and observed phenomenon. Starting over 25 years ago Heiles $(1979,1984,1990)$ detected large shell structures in Galactic neutral hydrogen, and in the intervening period a number of authors (e.g. Brinks 1981; Brinks \& Bajaja 1986; Deul \& den Hartog 1990; Puche et al. 1992; Kamphuis \& Sancisi 1993; Stavely-Smith et al. 1997; Wilcots \& Miller 1998; Walter \& Brinks 1999; Kim et al. 1999; Wills et al. 2002) have shown that such shells, often detected around holes in HI, are present in nearby gas rich galaxies. More recently McClureGriffiths et al. (2002) mapped hundreds of HI shells within the Galaxy, and Ehlerova \& Palous (2005) showed that in the outer galaxy these shells occupy a minimum of $5 \%$ of the disc volume. Although telescope resolution limits the minimum detectable size of an HI shell to some tens of pc even in nearby galaxies, many have been detected on scales of hundreds of pc, up to $1 \mathrm{kpc}$ in diameter.

Brinks and Bajaja showed that in M31 HI holes smaller than $300 \mathrm{pc}$ are correlated with OB associations, but no reliable correlations could be found for bigger holes. However, when sufficient spatial resolution can be applied, such as in the case of the SMC, it is notable that positional coincidence of the HI shell with a corresponding $\mathrm{OB}$ association occurs in only a minority of cases, as shown by Hatzidimitriou et al. (2005). These authors showed that of the 509 expanding HI shells catalogued in the SMC only some $40 \%$ are spatially correlated with OB associations, and some $10 \%$ lie in low density fields with no associated young stellar objects. Rhode et al. (1999) found evidence of young stellar clusters in only a small fraction of the HI holes in the dwarf galaxy Ho II. The age of the central stellar cluster located within the supergiant shell in the dwarf irregular galaxy IC 2574 is in agreement with the kinematic age of the shell, supporting the scenario in which the supernovae and stellar winds create the giant HI holes (Stewart \& Walter 2000).

Mechanisms of different kinds for producing large HI shells have been presented in the literature. The possibility that the infall of a high velocity cloud can give rise to HI holes and shells has been discussed by a number of authors with some quite detailed models (e.g. Tenorio-Tagle \& Bodenheimer 1988; Rand \& Stone 1996; Santillan et al. 1999; Murray \& Lin 2004). In the last two of these studies magnetic fields were also included. Another possibility, proposed by Hatzidimitriou et al. (2005) is a turbulent origin for at least some of these structures. The present article does not claim the scope to contrast those theoretical models with observations. All we will do is to present our scenario, based on observations of expanding $\mathrm{H} \alpha$ shells in $\mathrm{H}$ II regions, 
which offer a new quantitative element capable of contributing to explain the range of parameters observed for HI shells, including the largest of them.

The essential point of this article is to present evidence, using a set of observational data not hitherto applicable to this scenario (that of the presence and properties of expanding ionized shells within luminous H II regions) within an energetic framework of a set of models, that these ionized shells are the precursors of the previously observed class of neutral shells which have larger radii and lower expansion velocities. Although the scenario we present here is conventional in the sense that it links the HI shells to $\mathrm{OB}$ associations, our quantitative treatment is useful in showing that observations such as those of Hatzidimitriou et al. (2005) are in fact compatible with it. Typical measured velocities of HI shell expansion (e.g. Walter \& Brinks 1999), are of order $10 \mathrm{~km} \mathrm{~s}^{-1}$, much lower than the velocities detected in OB stellar winds (Herrero et al. 1992) and very much lower than velocities in supernova remnants (Weiler \& Sramek 1988; Chevalier 1977). The expansion velocities we find for the $\mathrm{H} \alpha$ shells are of order of $100 \mathrm{~km} \mathrm{~s}^{-1}$. In the context of our schematic model developed in this article these velocities are characteristic of the successive stages of the developing shell as it expands and does work against its surroundings. The timescales implied for a shell to reach radii of order $1 \mathrm{kpc}$ are at minimum a few times $10^{7} \mathrm{yr}$, which gives time for the stars in a coeval OB association to disappear. We also note that an expansion occurring in an anisotropic zone of the interstellar medium (ISM) will expand preferentially in the low density direction, so that an initiating star cluster would not lie close to its centre; for the largest shells this effect would be enhanced by Galactic differential rotation.

For these reasons we set out to detect the counterparts of HI shells at a much earlier stage, while they are more compact but expanding at higher velocities. Of course this implies that we cannot be looking at the same objects as those detected via the HI shells and holes, but rather at the $\mathrm{H}$ II regions which surround the $\mathrm{OB}$ associations and which mark the energetic impact of these associations on the ISM. To do this we used the optical technique most akin to $21 \mathrm{~cm}$ line mapping of $\mathrm{HI}$ : two dimensional emission line mapping in $\mathrm{H} \alpha$ with a Fabry-Perot interferometer. Some of these results have been reported previously (Relaño \& Beckman 2005a, Paper I), and showed the presence, within luminous $\mathrm{H}$ II regions, of dominant expansive components whose properties indicated that they could be the precursors of large HI shells. In Sect. 2 of the present article we summarize those observations, and describe further work of the same basic type on H II regions in the nearby galaxy NGC 5194 (M 51). In Sect. 3 we describe a very simple dynamical model in which the inputs from stellar winds and from supernovae are coupled to the surrounding gas, taking energy and momentum conservation as basic constraints. We apply this to our observed HII regions, using the measured $\mathrm{H} \alpha$ luminosities to estimate the massive stellar content used to calculate the energy and momentum inputs to the shells. We go on to compare these results with the range of energies and momenta in HI shells from the literature. In Sect. 4 we discuss general physical considerations relevant to modelling the implied evolutionary sequence and give our conclusions.

\section{Evidence for expanding shells in luminous HII regions}

Using galaxies whose populations of $\mathrm{H}$ II regions have published photometric catalogues in $\mathrm{H} \alpha$ (Rozas et al. 1996, 2000; Relaño et al. 2005b) we used complete intensity-velocity maps, also in $\mathrm{H} \alpha$, made with the TAURUS-II Fabry-Perot system on the $4.2 \mathrm{~m}$ WHT (Paper I) to make an exhaustive study of the integrated emission line profiles of the $\mathrm{HII}$ region population in three spiral galaxies: NGC 6951, NGC 3339 and NGC 1530. We found that more than one third of the regions in each galaxy showed high velocity low intensity peaks in the wings of the main emission lines. The fraction of the line profiles showing these wing features increased with the $\mathrm{S}: \mathrm{N}$ of the observations, so that, for example, NGC 1530, the galaxy with the highest S:N was the galaxy with the highest fraction of its profiles exhibiting wing features.

The interpretation we gave (Paper I) to these low intensity high velocity features is that they are evidence for supersonic expanding shells within the $\mathrm{H}$ II regions, and we could use their observational parameters to derive the properties of the shells. Fitting the features with Gaussians we could derive, first, the emission measure corresponding to the feature, which was used to derive the column density of a given shell and, using reasonable assumptions, go on to estimate its density (see Eq. (1) of Paper I). Secondly, the mean velocity separation between the central Gaussian emission component and the lower intensity wing features gave us the velocity of the expanding shell.

Here we have taken the dynamical parameters (mass and kinetic energy) of a sample of H II regions from Paper I. The sample was selected to cover the full range of $\mathrm{H} \alpha$ luminosities of the HII regions observed in the three galaxies (see Table 1). The estimated shell radii shown in Table 1 are rather crude estimates, since at the distances of the galaxies the linear resolution does not allow a clear measurement. Based on previous studies of expanding shells in extragalactic luminous $\mathrm{H}$ II regions much closer to the observer, 30 Doradus (Chu \& Kennicutt 1994) and NGC 604 (Yang et al. 1996), in Paper I we used a canonical shell radius of $R_{\text {shell }}=0.2 R_{\text {reg }}$ (i.e. $20 \%$ of the H II region radius) and a shell thickness of $4.5 \mathrm{pc}$. From these estimates we could go on to estimate the shell mass as a fraction of the total gas mass in the $\mathrm{H}$ II region. Average values for the $\mathrm{HII}$ regions in the three galaxies ranged from $20 \%$ for NGC 1530 to $26 \%$ for NGC 3359 , i.e. of order $20-25 \%$. We could then use the estimated mass and the measured velocity to derive an estimated kinetic energy of expansion for each shell. We obtained the equivalent number of $\mathrm{O} 3(\mathrm{~V})$ stars using the $\mathrm{H} \alpha$ luminosity of a given region and the value given by Vacca et al. (1996) for the ionizing photon output of an O3(V) star. We can later use the equivalent number to estimate the kinetic energy input from the stellar winds, using the estimate by Leitherer (1998) of the wind luminosity for an O3(V) star, integrated over the time the star is on the main sequence. This input energy is listed in the last column of Table 1. It is clear that we could have added the extra sophistication of looking at an IMF, instead of choosing a specific stellar spectral type, but the uncertainties implied would not have made this option a better one in terms of accurate quantification of the phenomena.

\subsection{Observations of NGC 5194}

We have followed the method used in Paper I and outlined in the paragraph above to study a sample of HII regions in the nearby galaxy NGC 5194 (M 51). The H $\alpha$ Fabry-Perot observations for this galaxy were obtained on the $1.6 \mathrm{~m}$ telescope of the Observatoire du Mont Mégantic (OMM) using the instrument FANTOMM (Hernandez et al. 2003; Gach et al. 2002). The final data cube consists of 48 spectral channels with a corresponding total integration time per channel of $5.16 \mathrm{~min}$. The channel resolution is $0.15 \AA$ (corresponding to $6.94 \mathrm{~km} \mathrm{~s}^{-1}$ ) and the effective 

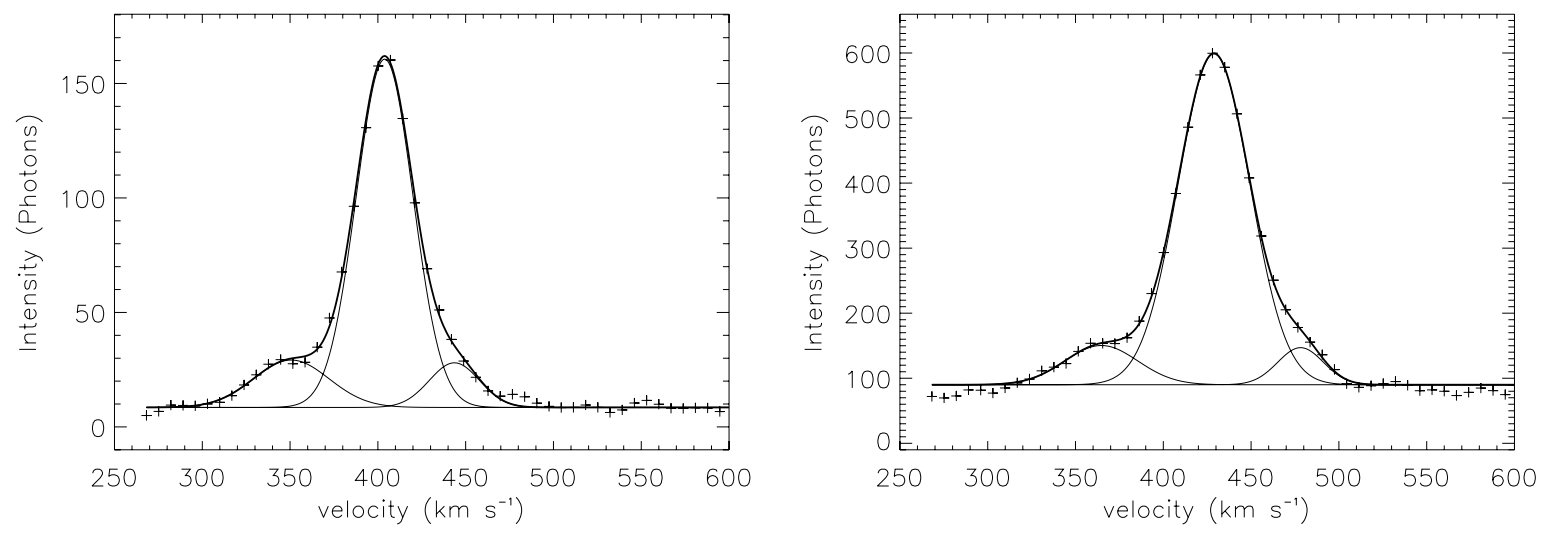

Fig. 1. Left: Emission line profile in $\mathrm{H} \alpha$ of the H II region 403 of M 51 in Rand's photometric catalogue. The profile was obtained from the FabryPerot data cube of the H II region. The Gaussian decomposition shown in the figure was obtained using the task PROFIT in the GIPSY package (van der Hulst et al. 1992). Right: Emission line profile of the H II region 312 of M 51 in Rand's catalogue obtained and fitted in the same way as in the left figure.

resolution of the observations is $R=18609$, which corresponds to $16 \mathrm{~km} \mathrm{~s}^{-1}$. The central wavelength of the redshifted narrow band blocking filter for $\mathrm{H} \alpha$ is $6581 \AA$, with a half width of $9.9 \AA$ corresponding to $\sim 450 \mathrm{~km} \mathrm{~s}^{-1}$.

The Photon Counting Camera had a scale of 1.6"/pixel and the seeing during the observations was $3-4{ }^{\prime \prime}$. Although this seeing cannot be considered as very good, the high spectral resolution allows us to study well the high velocity features in apertures commensurate with the seeing, which allows us to study the largest and most luminous $\mathrm{H}$ II regions adequately. A detailed description of the observations of NGC 5194 is given in Daigle et al. (2006a) and the data reduction is explained in more detail in Daigle et al. (2006b). The $\mathrm{H} \alpha$ luminosities for the H II regions in NGC 5194 were taken from a catalogue obtained by Rand (1992), and kindly supplied by the author. The radius of a region found in the catalog was used to estimate the highest aperture for the line profile of any given region under study.

In Fig. 1 we show two examples of line profiles for $\mathrm{H}$ II region 403 and 312 of M 51 in Rand's catalogue with the corresponding Gaussian components fitted to this spectrum. These figures clearly show the typical red and blue components called here wing features. For an extended sample of the line profiles from $\mathrm{H}$ II regions in other galaxies the reader is referred to Paper I. The results of the analysis for the emission line profiles of the HII region sample in NGC 5194 are shown in Table 1. For each H II region we extracted line profiles with apertures of increasing radius, and fitted them with the corresponding Gaussian components. The profile whose wing features yielded the best $\mathrm{S}: \mathrm{N}$ was taken as the representative spectrum of the $\mathrm{H}$ II region. The observational parameters of the wing features for each $\mathrm{H}$ II region do not vary significantly when increasing the aperture, which allows us to take the best $\mathrm{S}: \mathrm{N}$ line profile as the representative one for each $\mathrm{H}$ II region. The corresponding observational parameters, the Emission Measure and the velocities of the wing feature Gaussians with respect to the central emission peak were used to obtain the dynamical parameters of the shell for each region using the same criteria as those in Paper I. These parameters are listed in Table 1. The relative errors for the observational parameters are $0.2-0.3$ for the Emission Measures and $0.05-0.08$ for the velocity separation between the central and the shifted components. It is interesting to note that the absolute errors in the fitting procedure for the velocity of the shifted components are 2-3 $\mathrm{km} \mathrm{s}^{-1}$, much lower than the spectral resolution of the observations. This gives an idea of the validity of the Gaussian decomposition procedure.

\section{Energetics of the expanding shells}

\subsection{Starburst99 models}

Our aim here is to extrapolate from the properties of the shells we have observed within the $\mathrm{H}$ II regions to later times, taking the shells as representative, to show how their parameters would evolve with time and then to compare the extrapolated shells with HI shells observed in the Galaxy and nearby galaxies. In order to infer the energy inputs from the stars in the form of both stellar winds and supernovae, we have used from the literature the Starburst99 models (Leitherer et al. 1999). We take the case of an instantaneous starburst with a fixed stellar mass and integrate using two different Initial Mass Function (IMF) exponents, 1.3 and 2.3 for mass intervals $(0.1-0.5) M_{\odot}$ and $(0.5-100) M_{\odot}$, respectively. We take the conventional assumption that $8 M_{\odot}$ is the lower limit of a Zero Age Main Sequence star which will eventually explode as a supernova, and use the Padova evolutionary tracks at solar metallicity. Starburst99 then gives, from an initial time of $10^{4} \mathrm{yr}$ the (logarithmic) energy (in erg s${ }^{-1}$ ) input due to both stellar winds and supernovae as a function of time.

The principal input parameter in our Starburst99 models is the stellar mass associated with the burst, which was inferred from the $\mathrm{H} \alpha$ luminosity of the H II region, as described in Sect. 7.2.1 of Relaño et al. (2005b). We use the Salpeter IMF to estimate the ionizing mass of the star cluster and compare it to the mass estimate using an equivalent number of $\mathrm{O} 5(\mathrm{~V})$ stars. The normalizing factor obtained is then used when integrating the IMF within the appropriate mass limits $\left(0.1 M_{\odot}-100 M_{\odot}\right)$ and the total stellar mass in the cluster is derived. The output of Starburst 99 gives the energy produced by the stellar winds and supernovae within the star cluster as a function of the age of the cluster. We have taken the total energy produced by the stellar winds and also by the supernovae integrated over a time scale of $1.0 \times 10^{7} \mathrm{yr}$, and have studied the evolution of a shell over this time scale. Estimates of the ages of $\mathrm{H}$ II regions based on predicted ratios of emission line strength to continuum level (Copetti et al. 1985; Bresolin \& Kennicutt 1997) yield ages for $\mathrm{H}$ II regions in the range from $2 \mathrm{Myr}$ to $8 \mathrm{Myr}$, and show that the dynamical timescale adopted here is reasonable. 
Table 1. Shell parameters of a subsample of Hil regions in NGC 1530, NGC 3359, NGC 6951 and NGC 5194 . The shell parameters of the $\mathrm{H}$ II regions in the first three galaxies are from Paper I, while the rest are derived here. Column 1: number for the H II region in the catalogue of each galaxy. Column 2: logarithmic $\mathrm{H} \alpha$ luminosity. Column 3: shell radius. Column 4: measured shell expansion velocity. Column 5: shell electron density. Column 6: ionized mass of the shell. Column 7: kinetic energy of the shell. Column 8: combined kinetic energy of OB stellar winds, assuming an equivalent number of $\mathrm{O} 3(\mathrm{~V})$ type stars.

\begin{tabular}{cccccccc}
\hline \hline $\begin{array}{c}\text { Region } \\
\text { (number) }\end{array}$ & $\begin{array}{c}\log L_{\mathrm{H} \alpha} \\
\left(\mathrm{erg} \mathrm{s}^{-1}\right)\end{array}$ & $\begin{array}{c}R_{\text {shell }}(\mathrm{H} \alpha) \\
(\mathrm{pc})\end{array}$ & $\begin{array}{c}v_{\text {shell }}(\mathrm{H} \alpha) \\
\left(\mathrm{km} \mathrm{s}^{-1}\right)\end{array}$ & $\begin{array}{c}n_{\text {shell }}(\mathrm{H} \alpha) \\
\left(\mathrm{cm}^{-3}\right)\end{array}$ & $\begin{array}{c}M_{\text {shell }}(\mathrm{H} \alpha) \\
\left(10^{4} M_{\odot}\right)\end{array}$ & $\begin{array}{c}E_{\mathrm{K}}(\mathrm{H} \alpha) \\
\left(10^{51} \mathrm{erg}\right)\end{array}$ & $\begin{array}{c}E_{\text {wind }}(\mathrm{O} 3) \\
\left(10^{51} \mathrm{erg}\right)\end{array}$ \\
\hline NGC 1530 & & & & & & & \\
\hline 8 & 39.45 & 82.00 & 64.72 & 10.90 & 10.2 & 4.3 & 10.4 \\
22 & 39.12 & 60.92 & 49.38 & 9.37 & 4.9 & 1.2 & 4.9 \\
92 & 38.29 & 27.24 & 79.13 & 9.02 & 0.9 & 0.6 & 0.7 \\
\hline NGC 3359 & & & & & & & \\
\hline 6 & 39.15 & 69.62 & 63.97 & 8.36 & 5.7 & 2.3 & 5.3 \\
42 & 38.58 & 48.66 & 40.91 & 8.16 & 2.7 & 0.5 & 1.4 \\
92 & 38.19 & 36.38 & 51.98 & 4.80 & 0.9 & 0.2 & 0.6 \\
\hline NGC 6951 & & & & & & & \\
\hline 2 & 39.27 & 66.62 & 60.93 & 11.11 & 6.9 & 2.5 & 6.9 \\
18 & 38.67 & 48.66 & 47.62 & 6.50 & 2.2 & 0.5 & 1.7 \\
41 & 38.35 & 37.34 & 50.82 & 8.27 & 1.6 & 0.4 & 0.8 \\
\hline NGC 5194 & & & & & & & \\
\hline 403 & 38.94 & 63.93 & 46.75 & 8.96 & 5.1 & 1.1 & 3.2 \\
312 & 38.66 & 35.30 & 56.75 & 10.02 & 1.7 & 0.6 & 1.7 \\
416 & 38.46 & 52.48 & 50.80 & 4.08 & 1.6 & 0.4 & 1.1 \\
\hline
\end{tabular}

\subsection{Two basic driving mechanisms: stellar winds and supernova explosions}

There are two basic mechanisms which can drive the formation of a shell expanding into the ISM from an OB association: the stellar winds from young stars and the explosions of supernovae. The first mechanism was modelled when accounting for the shells observed in the $\mathrm{H}$ II region populations of the three spiral galaxies described in Paper I. We compared there the wind energy input from the equivalent numbers of O3(V) stars, derived using the $\mathrm{H} \alpha$ luminosity of a region, with the shell kinetic energy inferred from the kinematic observations. The conclusion was reached that shells with radii of up to $\sim 0.2 R_{\text {reg }}$ (where $R_{\text {reg }}$ is the radius of the $\mathrm{H}$ II region) could be quantitatively explained by the interaction of the stellar winds with the ISM, while some of the bigger shells located within the most luminous H II regions show kinetic energies which are too high to be explained as the result solely of wind interaction.

We have here gone on to improve the method used in Paper I, and now use the input energy from the stellar winds (here derived from the models implemented in Starburst99), but also add in the energy released in the subsequent supernovae. We are thus modelling the situation in which both mechanisms operate on the ISM surrounding a star cluster. The winds provide a continuous energy source which lasts for the stellar lifetime, i.e. $\sim 10^{6} \mathrm{yr}$, by this time an expanding shell has formed within the H II region. This is a single shell even if the stars "switch on" at dispersed times, because if the first star causes a shell to form, the wind from a second star will expand more quickly until it reaches the shell, and then couple its impulse to the shell, and this will occur for each subsequent star. Then, the supernovae occur inside the partially attenuated bubble produced by the winds. The situation has been described in some detail by Bruhweiler et al. (1980) and by Dyson (1981); the shock produced by the SN explosion sweeps up the material inside the cavity formed by the stellar winds, and impacts directly on the shell; then the strength of the transmitted shock is reduced by a factor which depends on the contrast in density between the cavity and the newly formed shell. The radiative cooling time within the shell can be very short, and then the momentum conservation is applied as the best approximation to describe the dynamics of the new formed shell.

The initial velocity is taken directly from the $\mathrm{H} \alpha$ observations, so that $v_{0}$ is the velocity of the shell as observed within an $\mathrm{H}$ II region, in $\mathrm{H} \alpha$ (Col. 4 of Table 1$)$. The radius at which the $\mathrm{SNe}$ explode $\left(R_{0}\right)$, is taken to be the radius of a wind blown shell. This is estimated applying the model of Dyson (1980) for the adiabatic expansion of a stellar wind bubble. The inputs required in this model are the energy flux of the stellar winds, the initial gas density, and the time during which the stellar winds act. Taking the stellar wind energy from Starburst99 models we apply the equations of the adiabatic model to a range of values of $n$ and $t_{0}$. The optimum combination of these variables for producing the measured parameters of the wind blown portion of the shell expansion is $n=0.5 \mathrm{~cm}^{-3}$ and $t_{0}=10^{6} \mathrm{yr}$. Using these values the shell radius is found to be $\sim 0.3 R_{\text {reg }}$ and the velocities just slightly lower than the observed $\mathrm{H} \alpha$ velocities. In the end we chose to use $R_{0}=0.5 R_{\text {reg }}$ for the initial radius of the shell in the post SN phase of our modelling. This is the shell radius at which the $\mathrm{SNe}$ explode and although it is a little higher than the radius derived in the adiabatic model, it allows us to simplify our modelling by separating the adiabatic expansion phase due to the winds from the momentum conserving phase after the supernovae have exploded. We used an ambient interstellar density of $n=0.1 \mathrm{~cm}^{-3}$, slightly lower than the value estimated from the adiabatic model. This values is chosen as a practical compromise which takes partially into account that the density decreases with distance from the plane of the galaxy, a circumstance of relevance especially for the larger HI shells. We confirmed that using a density of $0.5 \mathrm{~cm}^{-3}$ changes the magnitude of the results by a relative modest fraction (slightly $(\sim 20 \%)$ higher radii and higher velocities $(\sim 13 \%))$.

Using the model assumptions described above we start with the shell whose properties we have derived from our $\mathrm{H} \alpha$ observations and run the models adding the supernova inputs as explained. We assume that the stellar winds have acted over a time interval of $t_{\mathrm{o}}=10^{6} \mathrm{yr}$ and that at this time the supernovae explode with no time intervals between them. The supernovae then sweep up the material within the bubble and push outwards the 

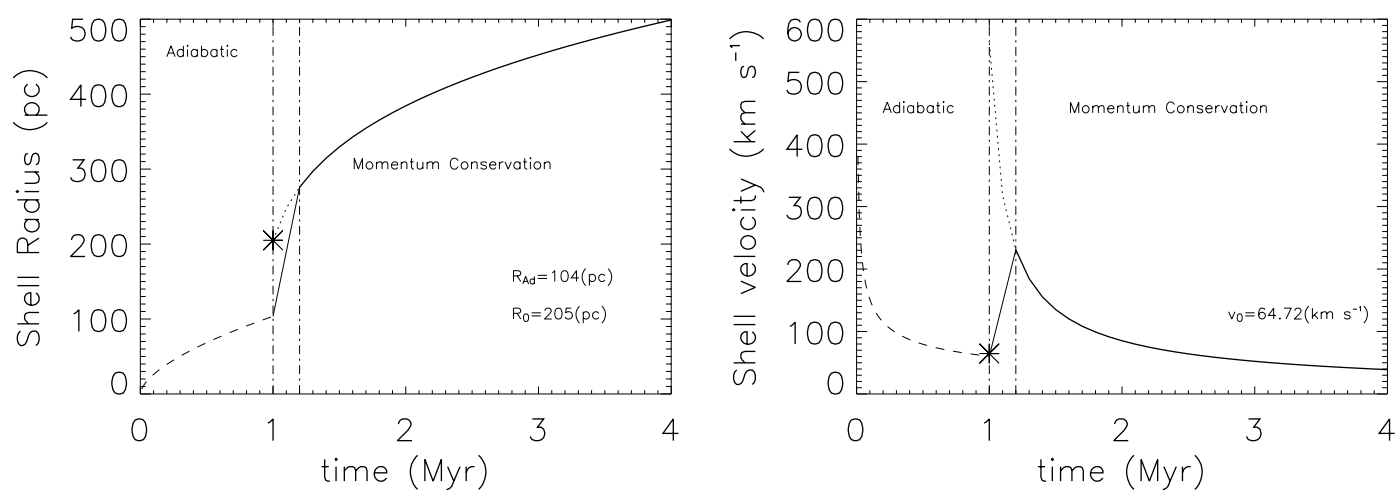

Fig. 2. Left. Evolution of the shell radius of the adiabatic and momentum conserving phases for the parameters shown by H II region 8 of NGC 1530 . $R_{\text {Ad }}$ is the initial radius found from the adiabatic model, which predicts in general shell radii of $0.3 R_{\text {reg }}$, and velocities comparable with those observed for the ionized shells within the H II regions. $R_{0}$ is the input radius for the momentum conserving phase. We have not explicitly modelled the transition between the phases, and this is represented schematically here by the line joining the two curves in the time interval between 1 and 1.2 Myr. Right. Evolution of the shell velocity for the same example H II region. The shell velocity predicted in the adiabatic phase is close to the observed velocity of the $\mathrm{H} \alpha$ shell (see Col. 4 of Table 1). In our simplified model of the momentum conserving phase, in which the SN input is instantaneous, the initial velocity would be boosted to the unrealistically high value indicated in the figure. A schematic representation of the transition is given by the line joining the two curves in the time interval between 1 and $1.2 \mathrm{Myr}$. Although the assumption of instantaneous energy injection is too simple, the overall energetics of the model, which predicts a final HI radius of $653.5 \mathrm{pc}$ and a corresponding $\mathrm{HI}$ expansion velocity of $17 \mathrm{~km} \mathrm{~s}^{-1}$ (see Table 2) are physically reasonable.

shell created by the stellar winds. The application of momentum conservation gives the radius and the shell velocity as a function of time according to the expressions (Dyson 1980; Bruhweiler et al. 1980):

$R_{\text {shell }}(t)=\left[R_{0}^{4}+\frac{3 M_{0} v_{0}}{n m_{\mathrm{H}} \pi}\left(t-t_{0}\right)\right]^{(1 / 4)}$

$v_{\text {shell }}(t)=R_{\text {shell }}^{-3} \frac{3 M_{0} v_{0}}{4 n m_{\mathrm{H}} \pi}$

where $R_{0}$ and $v_{0}$ are the radius and velocity of the expanding shell at the switch-on time of the supernovae, $n$ is the ambient interstellar density and $M_{0}$ is the mass swept up by the effect of the supernova explosions. In Fig. 2 we show the time evolution of the shell radius and shell velocity for the $\mathrm{H}$ II region 8 in NGC 1530 (first raw of Table 1). The figure, that can be taken as a characteristic example, shows qualitatively how the shell forms in the wind blown phase of the expansion. It then receives a boost from the supernova input which initially accelerates it, but the velocity is subsequently reduced as the shell picks up mass from the surrounding ISM and expands reaching a radius within the range measured for HI supershells.

The mass $M_{0}$ in Eq. (2) is the total mass contained within the bubble at the initial radius $R_{0}$, which is assumed to be completely swept up as a consequence of the SN explosions. To obtain the mass within the bubble we have used the method to derive the mass of gas in an H II region described in Relaño et al. (2005b), where we included a factor to take into account the neutral gas not observed directly in $\mathrm{H} \alpha$ (see Giammanco et al. 2004). We then apply Eqs. (1) and (2) and compute a "final" radius for the shell, $R_{\text {shell }}$, and a "final" velocity, $v_{\text {shell }}$, at a time of $1.0 \times 10^{7} \mathrm{yr}$, which agrees with the dynamical age range of the suppershells observed in HI (McClure-Griffiths et al. 2002; Walter \& Brinks 1999). An integration of the models at a time of $0.5 \times 10^{7} \mathrm{yr}$ will produce velocity values between $15-30 \mathrm{~km} \mathrm{~s}^{-1}$, while at longer times $\left(5.0 \times 10^{7} \mathrm{yr}\right)$ the velocities will be $\sim 3 \mathrm{~km} \mathrm{~s}^{-1}$, much lower than those shown here. These last values are not expected to have a clearly defined physical meaning, since the shell loses its identity at $v \sim 5 \mathrm{~km} \mathrm{~s}^{-1}$ (Dyson 1981), and for the corresponding radii, of order $500 \mathrm{pc}$ of greater, a shell will tend to be disrupted by global galactic effects such as differential rotation. We present the results of the models in Table 2, where we also show the total combined input energies of stellar winds and supernovae from Starburst99, and the kinetic energy of the shell obtained using the standard expression $E_{\mathrm{k}}=\frac{1}{2} m_{\mathrm{HI}} v_{\mathrm{HI}}^{2}$. An interesting supplementary exercise was to run models in which a second burst of star formation boosted the injection of energy into the expanding shells. According to the time at which the model output is examined this produces HI shells either with a range of radii similar to those observed but with a velocity range substantially higher, or with a range of velocities similar to those observed but with a range of radii substantially higher. This suggests strongly that the stars in $\mathrm{OB}$ associations are coeval. In the next section we compare our results with a sample of HI shells observed in two different galaxies.

\subsection{Comparison with $\mathrm{HI}$ observations}

We have selected a representative sample of HI shells with different sizes observed by Walter \& Brinks (1999) in the dwarf galaxy IC 2574 and by McClure-Griffiths et al. (2002) in the Milky Way. We have limited our sample to those shells classified by Walter \& Brinks as "classic holes" and we have avoided "merged shells" and chimneys in the McClure-Griffiths et al. data set. Our results in Table 2 show good broad agreement with the parameters of the observed HI shells reported in the two articles cited. The modelled range of shell velocities, $v_{\text {shell }}=$ (8-17) $\mathrm{km} \mathrm{s}^{-1}$, falls nicely within the range of the observed sample, $v_{\text {shell }}=(4-21) \mathrm{km} \mathrm{s}^{-1}$. The range of shell masses produced in the models, $M_{\text {shell }}=(3-29) \times 10^{5} M_{\odot}$ is within the range of masses observed by McClure-Griffiths et al. (2002), $(1-44) \times 10^{5} M_{\odot}$, but is somewhat higher than the range found by Walter \& Brinks (1999), $(0.02-18) \times 10^{5} M_{\odot}$. The first study yields values for the HI number density $0.5-1.7 \mathrm{~cm}^{-3}$, while the second study takes the scale height of the galaxy disk into account (the galaxy is in any case much smaller) and finds values in the range $0.05-0.17 \mathrm{~cm}^{-3}$. The smaller values for the range of the basic parameters in Walter \& Brinks (1999) can 
Table 2. Energetics of the HI predicted shells for the sample of H II regions in NGC 1530, NGC 3359, NGC 6951 and NGC 5194. The stellar winds act over $10^{6} \mathrm{yr}$, the time at which the $\mathrm{SNe}$ explode, the final integration time of the models is $10^{7} \mathrm{yr}$. Column 1: $\mathrm{H}$ II region number. Column 2: shell radius. Column 3: shell expansion velocity. Column 4: mass of the shell. Column 5: energy input from the supernovae and stellar winds. Column 6: kinetic energy of the shell. Column 7: efficiency of the SN to convert their energies into kinetic energy in the ISM.

\begin{tabular}{ccccccc}
\hline \hline $\begin{array}{c}\text { Region } \\
(\text { number })\end{array}$ & $\begin{array}{c}R_{\text {shell }} \\
(\mathrm{pc})\end{array}$ & $\begin{array}{c}v_{\text {shell }} \\
\left(\mathrm{km} \mathrm{s}^{-1}\right)\end{array}$ & $\begin{array}{c}M_{\text {shell }} \\
\left(10^{5} M_{\odot}\right)\end{array}$ & $\begin{array}{c}E_{(\mathrm{SW}+\mathrm{SN})} \\
\left(10^{52} \mathrm{erg}\right)\end{array}$ & $\begin{array}{c}E_{\mathrm{K}}(\mathrm{shell}) \\
\left(10^{51} \mathrm{erg}\right)\end{array}$ & $\begin{array}{c}\text { Efficiency } \\
(\%)\end{array}$ \\
\hline $1530-8$ & 653.5 & 17.3 & 28.9 & 36.1 & 8.6 & 3.7 \\
$1530-22$ & 505.8 & 13.4 & 13.4 & 16.8 & 2.4 & 2.2 \\
$1530-92$ & 351.6 & 9.4 & 4.5 & 2.7 & 0.4 & 2.3 \\
\hline $3359-6$ & 550.2 & 14.6 & 17.2 & 18.1 & 3.7 & 3.2 \\
$3359-42$ & 353.5 & 9.3 & 4.6 & 4.7 & 0.4 & 1.3 \\
$3359-92$ & 299.7 & 8.0 & 2.8 & 2.0 & 0.2 & 1.4 \\
\hline $6951-2$ & 579.4 & 15.4 & 20.1 & 24.1 & 4.8 & 3.1 \\
$6951-18$ & 386.8 & 10.3 & 6.0 & 6.0 & 0.6 & 1.6 \\
$6951-41$ & 326.9 & 8.7 & 3.6 & 2.7 & 0.3 & 1.6 \\
\hline $5194-403$ & 449.7 & 11.9 & 9.4 & 10.5 & 1.3 & 2.0 \\
$5194-312$ & 400.9 & 10.7 & 6.7 & 5.5 & 0.8 & 2.1 \\
$5194-416$ & 348.8 & 9.2 & 4.4 & 3.7 & 0.4 & 1.5 \\
\hline
\end{tabular}

be ascribed to the fact that they were observing a dwarf galaxy where the range of ISM densities is lower, and this will necessarily be reflected in the ranges of shell mass and kinetic energy. Our H II region observations have all been taken from regions in essentially Milky Way mass galaxies. The better agreement between the range of parameters derived by applying dynamical modelling to shells observed in these regions, using the appropriate values of mass, velocity and ISM density, and the HI shells observed in the Milky Way by McClure-Griffiths et al. is not fortuitous.

\section{Final remarks}

It is not surprising to find expanding shells around OB associations, but a variety of scenarios have been proposed in which these can arise. OB stars have a major dynamical impact on their surroundings, which can take four forms: the direct influence of ionizing radiation on the surrounding gas, the influence of nonionizing radiation on the surrounding gas, mediated by its dust content, the effect of the outflow of gas from the stars themselves in the form of stellar winds, and the effects of the violent outflow of gas in supernova explosions. Our underlying concepts here are based on the seminal article by Dyson (1981), who considered the dynamical effects of stellar winds on $\mathrm{H}$ II regions in the necessary presence of the ionizing radiation which forms them.

In treating the development of the wind-blown shells we must then take into account that on a well determined timescale there will be an additional dynamical input from the supernovae in the OB association. The timescale is of key importance, because during the main sequence lifetime of an $\mathrm{O}$ star, of order $10^{6} \mathrm{yr}$, a wind-blown shell expanding at a characteristic velocity of $\sim 50 \mathrm{~km} \mathrm{~s}^{-1}$ will reach a radius of $\sim 50 \mathrm{pc}$. This implies that the supernovae, which explode in the star cluster will at first expand very rapidly into a low density environment cleared by the winds, but will then impinge on the denser region marked by the shell and the surrounding ISM. Under these circumstances the adiabatic phase (Sedov 1959; Chevalier 1977) of the SN remnant expansion will immediately give way to the snowplough phase (Chevalier 1974) and the dynamical coupling of the SN remnant to the expanding shell will be determined by momentum conservation. We find that a typical fraction of the expansion energy of the SN remnant transferred to the shell will be $(1-4) \%$ (see last column in Table 2), with the rest either taken up as turbulence or radiated away. Further observations and more complete analysis would be necessary in order to set more rigorous constraints on the efficiencies under a variety of conditions. For example, if the largest bubbles blow out when expanding into lower gas densities, the efficiency of the process will be predictably lower than those found here.

To summarize, the detection and measurement of the physical parameters of dominant expanding shells within a major fraction of highly luminous extragalactic giant $\mathrm{H}$ II regions presented in Paper I and fully confirmed by the observational results in the present article, has given us a starting point from which to present a scenario relating the presence of these shells to the presence and the properties of the much larger HI shells detected in the Milky Way and in nearby galaxies. The result of applying a straightforward model embodying this scenario is that the HI shells in general most probably had $\mathrm{H} \alpha$ shells as their precursors. The time scales within the model are such that one should not expect to observe both the precursor shell and the HI shell for the same system, so that our results are necessarily statistical in nature. However, we can conclude that the observations of Hatzidimitrou et al. (2005), who observed stars in OB associations at the centres of only a minority of their HI shells, are fully compatible with our scenario. A suggestive, though perhaps not conclusive secondary inference, is that the stars in an OB association probably form in a single group not dispersed in formation epoch.

Acknowledgements. This work has been supported by the Spanish Ministry of Education and Science within the PNAYA (Spanish National Programme for Astronomy and Astrophysics) via projects AYA2004-08251-CO2-01/02, and ESP2003-00915, and by project P3/86 of the Instituto de Astrofísica de Canarias. We thank Richard Rand for kindly providing us with the H II region catalogue of NGC 5194. We are grateful to John Dyson for valuable discussions on theoretical aspects of this work. We thank the second anonymous referee for helpful comments and suggestions which enabled us to improve the article.

\section{References}

Bresolin, F., \& Kennicutt, R. C. Jr. 1997, AJ, 113, 975

Brinks, E. 1981, A\&A, 95, 1

Brinks, E., \& Bajaja, E. 1986, A\&A, 169, 14

Bruhweiler, F. C., Gull, T. R., Kafatos, M., \& Sofia, S. 1980, ApJ, 238, L27

Chevalier, R. A. 1974, ApJ, 188, 501

Chevalier, R. A. 1977, ARA\&A, 15, 175

Chu, Y.-H., \& Kennicutt, R. C. 1994, ApJ, 425, 720

Copetti, M. V. F., Pastoriza, M. G., \& Dottori, H. A. 1985, A\&A, 152, 427

Daigle, O., Carignan, C., Amram, P., et al. 2006a, MNRAS, 367, 469

Daigle, O., Carignan, C., Hernandez, O., Chemin, L., \& Amram, P. 2006b, MNRAS, 368, 1016 
Deul, E. R., \& den Hartog, R. H. 1990, A\&A, 230, 153

Dyson, J. E. 1980, Physics of the Interstellar Medium, (New York: John Wiley \& Sons), 145

Dyson, J. E. 1981, in Investigating the Universe, F. D. Kahn, ed. D. Reidel, Dordrecht, 125

Ehlerova, S., \& Palous, J. 2005, A\&A, 437, 101

Gach, J.-L., Hernandez, O., Boulesteix, J., et al. 2002, PASP, 114, 1043

Giammanco, C., Beckman, J. E., Zurita, A., \& Relaño, M. 2004, A\&A, 424, 877

Hatzidimitriou, D., Stanimirovic, S., Maragoudaki, F., et al. 2005, MNRAS, 360, 1171

Heiles, C. 1990, ApJ, 354, 483

Heiles, C. 1984, ApJS, 55, 585

Heiles, C. 1979, ApJ, 229, 533

Hernandez, O., Gach, J., Carignan, C., \& Boulesteix, J. 2003, SPIE, 4841, 1472

Herrero, A., Kudritzki, R. P., Vilchez, J. M., et al. 1992, A\&A, 261, 209

Kamphuis, J. J., \& Sancisi, R. 1993, A\&A, 273, 31

Kim, S., Dopita, M. A., Staveley-Smith, L., \& Bessell, M. S. 1999, AJ, 118, 2797

Leitherer, C. 1998, Stellar Astrophysics for the Local Group, ed. A. Aparicio, A. Herrero, \& F. Sánchez (Cambridge: Cambridge University Press), 527

Leitherer, C., Schaerer, D., Goldader, J. D., et al. 1999, ApJSS, 123, 3

McClure-Griffiths, N. M., Dickey, J. M., Gaensler, B. M., \& Green, A. J. 2002, ApJ, 578, 176

Murray, S., \& Lin, D. 2004, ApJ, 615, 586

Puche, D., Westpfahl, D., Brinks, E., \& Roy, J.-R. 1992, AJ, 103, 1841
Rand, R. J. 1992, AJ, 103, 815

Rand, R. J., \& Stone, J. M. 1996, AJ, 111, 190

Rhode, K. L., Salzer, J. J., Westpfahl, D. J., \& Radice, L. A. 1999, AJ, 118, 323

Rozas, M., Beckman, J. E., \& Knapen, J.-H. 1996, A\&A, 307, 735

Rozas, M., Zurita, A., \& Beckman, J. E. 2000, A\&A, 354, 823

Relaño, M., \& Beckman, J. E. 2005a, A\&A, 430, 911 (Paper I)

Relaño, M., Beckman, J. E., Zurita, A., Rozas, M., \& Giammanco, C. 2005b, A\&A, 431, 235

Santillan, A., Franco, J., Martos, M., \& Kim, J. 1999, ApJ, 515, 657

Sedov, L. 1959, Similarity and Dimensional Methods in Mechanics (New York: Academic)

Staveley-Smith, L., Sault, R. J., Hatzidimitriou, D., Kesteven, M., \& McConnell, D. 1997, MNRAS, 289, 225

Stewart, S. G., \& Walter, F. 2000, ApJ, 120, 1794

Tenorio-Tagle, G., \& Bodenheimer, P. 1988, ARA\&A, 26, 145

Yang, H., Chu, Y.-H., Skillman, E. D., \& Terlevich, R. 1996, AJ, 112, 146

Vacca, W. D., Garmany, C. D., \& Shull, M. 1996, ApJ, 460, 914

van der Hulst, J. M., Terlouw, J. P., Begeman, K. G., Zwitser, W., \& Roelfsema, P. R. 1992, Astronomical Data Analysis Software and Systems I, ed. D. M. Worrall, C. Biemesderfer, \& J. Barnes, ASP Conf. Ser., 25, 131

Weiler, K. W., \& Sramek, R. A. 1988, ARA\&A, 26, 295

Wilcots, E. M., \& Miller, B. W. 1998, AJ, 116, 2363

Wills, K. A., Pedlar, A., \& Muxlow, T. W. B. 2002, MNRAS, 331, 313

Walter, F., \& Brinks, E. 1999, AJ, 118, 273 